\title{
Post Infarction left ventricle remodeling surgical treatment, different ways to get same result
}

\author{
Lorenzo Menicanti ${ }^{1}$ \\ ${ }^{1}$ IRCCS Policlinico San Donato
}

November 15, 2021

\begin{abstract}
The surgical ventricular restoration is an evolution of treatment of left ventricle aneurysm. The aetioloy of left ventricle aneurysm and the dilated post AMI cardiomiopaty is the same; the difference is in the extension of scarred tissue and in the quality of remote zone. Because in this anatomical situation the geometry of left left ventricle can be deeply affected, it can very difficult to have point of reference as position of apex or papillary mussles. Using a sizer and combine different surgical thecniques allow to rebuilt a ventricle with appropriate volume and shape.
\end{abstract}

COMMENTARY:

Post Infarction left ventricle remodeling surgical treatment, different ways to get same result

Lorenzo A. Menicanti, MD

IRCCS Policlinico San Donato Hospital, Milan, Italy

Editorial commentary on an article entitled: An historical appraisal of the techniques of left ventricular volume reduction in ischemic cardiomyopathy: who did what?

No conflict of interest exists

Funding statement: none

Total words: 1198

Address for Correspondence:

Lorenzo A. Menicanti, MD

IRCCS Policlinico San Donato

Head of Cardiac Surgery Department

Scientific Director

Via Morandi 30 - 20097 San Donato Milanese

Milan, Italy

Email: lorenzo.menicanti@grupposandonato.it

Phone + 3952774514 - 4521

Fax +3952774327 
The surgical treatment of left ventricle aneurysm is a well-established procedure and the guidelines of the treatment of coronary artery disease consider the procedure with an evidence IIA 1.

The story of this procedure started long time ago when Beck2 in 1944 reduced the volume of a left ventricle aneurysm with a fascia lata patch, secured to the pericardium; the patch is plicate in length bringing near the borders of the pericardium, in this way the dilatation of the left ventricle stuck to the pericardium is reduced.

Calafiore and coworkers3, in this issue of Journal of Cardiac Surgery, describe the evolution of treatment from Beck's first attempt to more recent techniques, using Extracorporeal Circulation and Cardioplegic arrest, non-available at Beck's time.

Calafiore describes in a complete and elegant way the different techniques performed to achieve the same result: rebuild a left ventricle cavity with dimension and shape as more physiological as possible. All major contributions are described.

Correctly, there is no mention of Batista 4 procedure; this procedure was proposed for patients with very large left ventricle and increased wall thickness determined mainly by Chagas disease. In this pathological situation, the outcomes were positive; in opposite when ischemic patient were treated with this technique, the results were poor. This could have been expected: the Batista procedure resects a portion of lateral wall. When a dilatation of left ventricle, after AM,I is present, in majority of cases, is due to an occlusion of left anterior descending coronary. The anteroseptal portion of the left ventricle is scarred and the lateral wall is one of the functioning parts of left ventricle. If a portion is resected, the volume is reduced but it can harm the contractile function of lateral wall.

After an Acute Myocardial Infarction, involving the LAD territory, it is possible that the scar tissue increases in extension, producing a classical left ventricle aneurism with a well-defined neck and a transitional zone between the scar and normal contractile myocardium with normal wall thickness.

However, when the scar extension involves a larger area in the antero-septal-apical region, the classical phenotype of left ventricle aneurism with a well-defined neck dividing the contractile basal portion from the akinetic or diskinetic anteroseptal apical region, is not present anymore. This anatomical presentation cannot be described as Left Ventricle Aneurysm but rather as an Ischemic post-AMI Cardiomyopathy5. The pathology is of course the same but the effects on the function and shape of ventricle are more complex and detrimental.

Nowadays this clinical presentation is more frequent because of the wide use of primary PCI, aggressive medical therapy and PCI performed very frequently in follow-up period of these patients.

All these treatments reduce the mortality of AMI, but increase the number of patients with an enlarged left ventricle and heart failure.

In case of a primitive cardiomyopathy, the thickness and the contractility of left ventricle wall is reduced in all segment in a homogenous way, differently from a Left Ventricle Aneurysm or dilated ischemic post AMI cardiomyopathy, where, segments have different thickness and function: reduced in scarred zone and near normal in remote zone. The success of the left ventricle reconstruction procedure depends on the extension and function of the myocardium far from the infarcted area6.

In these pathophysiological conditions, the wall stress induced by the increased cavity volume, increases oxygen consumption and decreases the contractile reserve mainly in normal functioning part of the ventricle. Consequently, the reduction of left ventricle volume leads to a reduction of wall stress, decreasing oxygen demand and increasing contractile reserve. The reduction of heart failure markers after Surgical Ventricular Restoration is the demonstration of this positive effect7.

The shape of the ventricle is important: an ellipsoid shaped ventricle has a better mechanical resynchronization and avoiding sphericalizazion improves the function of mitral valve and decreases the risk of diastolic disfunction. 
Surgical Ventricular Restoration or Reconstruction before being a surgical technique is a clear Concept. The Surgical Procedure to be effective has to achieve:

a) Effective Left Ventricle Volume Reduction (less than $60 \mathrm{ml} / \mathrm{m} 2 \mathrm{ESVI}$ )

b) Sphericity Index in normal range avoiding value below 0.6

c) Complete coronary revascularization

All surgical techniques achieving these targets are good treatments regardless the authors and modifications.

The main differences between techniques consist in using a sizer to define precisely the target volume and the use of a prosthetic patch to rebuild the left ventricle cavity.

The baseline characteristics of ventricle can determine the final shape and volume.

There are some situations very favorable; in which the base of dilatation is small and the longitudinal diameter is preserved. Closing the base in every way, (purse string, small patch, linear closure) comfortable for the surgeon brings a good result but unfortunately similar situation is very rare.

Conversely, let assume to have a big ventricle with a large base parallel to the plane of mitral valve with reduction of basal to apex diameter, in this situation not all techniques can achieve the same result, with the possibility to determine a sphericalizazion with an incorrect control of the volume.

When an occlusion of the LDA produces a severe dilatation of antero-septal portion of the left ventricle, the dilatation can involve not only the apex of ventricle but also, extending around the apex, the inferior wall. In this situation, the transitional zone is almost at the base of papillary muscles.

Calafiore describes very similar situation in fig. 4 and 6 of his paper.

In Fig. 4 the inferior dilatation is unchanged, in Fig. 6 it is evident the important reduction of longitudinal diameter of the left ventricle.

A sizer, chosen according to Body Surface Area of patients, inserted in the cavity of left ventricle gives to surgeon two information: the position of the new apex, keeping in the normal range the longitudinal diameter and the target volume8.

Scar tissue is often present between the transitional zone very near to the base of papillary mussels, and the position of the new apex pointed by the sizer. A plication of the scarred wall can be performed starting from the base of the papillary and reaching the apex of sizer. In the way the inferior dilatation is obliterated, this plication with a very short suture, less than two centimeters, allows to respect the longitudinal diameter of left ventricle, avoiding sphericalizazion.

The sizer indicate also the target volume; the cavity can be closed with a linear suture over the sizer bringing the anterior wall against the septum, and excluding from the cavity the scar tissue of the septum in the same way described in Fig. 3 in Calafiore paper.

After an AMI, Surgical Ventricular Reconstruction is a very powerful tool in the hands of surgeons for restoring a ventricle with volume and shape very close to normal. The exclusion of scar tissue from the left cavity reduces left ventricle volume, reaching the target of $60 \mathrm{ml} / \mathrm{m} 2$ ESVI. The use of sizer allows a better reconstruction, pointing out the correct position of the new apex. The result will be a ventricle of targeted volume and physiological ellipsoidal shape. All types of phenotypes dilatation can be approached avoiding the use of a patch to get a shape with a sphericity index near to normal.

References

1. Neumann FJ, Sousa-Uva M, Ahlssom A, et al; 2018 ESC/EACTS Guidelines on myocardial revascularization: The Task Force on Myocardial Revascularization of the European Society of Cardiology 
(ESC) and the European Association for Cardio-Thoracic Surgery (EACTS) Developed with the special contribution of the European Association of Percutaneous Cardiovascular Interventions (EAPCI). Eur Heart J. 2018; 00:1-96

2. Beck CS. Operation for Aneurysm of the Heart. Ann Surg 1944; 120:34-40.

3. Calafiore AM, Totaro A, Prapas S, et al. An historical appraisal of the techniques of left ventricular volume reduction in ischemic cardiomyopathy: who did what? Journal of Cardiac Surgery 2021(in press)

4. Batista RJ, Verde J, Neri P, et al. Partial left ventriculectomy to treat end-stage heart disease. Ann Thorac Surg 1997; 64: 634-38.

5. Menicanti L, Castelvecchio S, Left ventricular reconstruction. In Mastery of Cardiothoracic Surgery, by Larry Kaiser, Irving Kron, and Thomas Spray - Third edition 2014; 54: 519-31.

6. Castelvecchio S, Careri G, Ambrogi F, et al. Myocardial scar location as detected by cardiac magnetic resonance is associated with the outcome in heart failure patients undergoing surgical ventricular reconstruction. Eur J Cardiothorac Surg. 2018; 53:143-49.

7. Castelvecchio S, Baryshnikova E, Pina IL, et al. Longitudinal profile of NT-proBNP levels in ischemic heart failure patients undergoing surgical ventricular reconstruction: The Biomarker Plus study. Int J Cardiol. 2018; 260: 24-30.

8. Castelvecchio S, Pappalardo OA, Menicanti L, Myocardial reconstruction in ischaemic cardiomyopathy, Eur J Cardio-Thoracic Surg. 55 (2019) i49-i56. 